\title{
Retrospective comparison of a novel-triple row to modified mason allen rotator cuff repair in a worker's comp population
}

\begin{abstract}
Introduction: As the number of rotator cuff repairs increases there is a search for the best surgical approach. Clinical outcome measures fail to distinguish the benefit of various repair procedures.

Methods: A novel triple row (NTR) repair that increases suture passes through the tendon is compared to a modified Mason Allen (MMA) repair in a worker's comp population. Outcome measures are forward flexion and external rotation measured in degrees and external strength with supraspinatus isolation. NTR repairs were used for larger tendon tears relative to those repaired with MMA.

Results: Outcome results were comparable for the two repair techniques despite average tear area being $3.87 \mathrm{~cm}^{2}$ for NTR and $1.35 \mathrm{~cm}^{2}$ for MMA repairs. Retear rates were $3.4 \%$ and $15 \%$ for NTR repairs.

Discussion: NTR repair focuses on tendon footplate restoration. Outcome measures for RCRs using either MMA or NTR procedures were essentially the same despite the fact that tear size in the NTR group was significantly larger. When the tear sizes were comparable for MMA and NTR for males, all the performance measures were improved for NTR and in several instances were somewhat better than for MMA repairs. Conclusion: NTR repair was accomplished using 3 generic anchors at a considerable cost savings. Based on clinical and biomedical outcomes of NTR repair it is recommended that large tears be repaired with multiple suture passes through medial to lateral positions. NTR repair results in small gap formation, high ultimate failure load, and significant cost savings.
\end{abstract}

Volume 3 Issue I - 2015

\author{
Blair A Rhode, William S Rhode \\ Orland Park Orthopedics, USA
}

Correspondence: Blair A Rhode, Orland Park Orthopedics, I6450 S 104th AV, Orland Park, Illinois 60467, USA, Tel 708-364844I,Email Blairbones@gmail.com

Received: June 29, 2015 | Published: July 07, 2015

Keywords: rotator cuff, workers comp, triple row, generic anchors

Abbreviations: RC, rotator cuff; RCR, rotator cuff repair; NTR, novel triple row; MMA, modified mason allen; SR, single row; DR, double row; PEEK, polyetheretherketone; UHMWPE, ultra-high molecular weight polyethylene; SD, standard deviation; RōG, Rhode orthopedic group

\section{Introduction}

Rotator cuff ( $\mathrm{RC}$ ) repairs employ suture-anchor combinations in a variety of configurations. The configuration that results in the best clinical outcome remains debated. Outcomes are influenced by tear size, patient age, obesity and exposure intensity. ${ }^{1-4}$ Between 1996 and 2006 rotator cuff repairs have increased $141 \%$ and arthroscopic repairs increased $600 \% .^{5}$ The goal of rotator cuff repair (RCR) is tendon-tobone fixation with a minimal gap formation that is maintained long enough for healing to take place. Despite numerous clinical studies that compared single-row (SR) to double-row (DR) suture-anchor repairs, subjective clinical outcomes have been essentially the same for the two repair techniques. Patients appear to be equally satisfied irrespective of the RCR technique. ${ }^{6-11}$

One outcome measure that presents a clear difference is the retear rate of RCRs. A review of seven RCR studies of SR vs DR repairs reports SR retear rate is approximately twice the DR retear rate. ${ }^{9} \mathrm{~A}$ recent study reported a healing rate of $93 \%$ for a DR repair and $74.1 \%$ for a SR repair with the SR to DR retear ratio being 3.57. ${ }^{12}$ A major factor in RCR outcomes is the initial tear size because massive tear repairs result in poorer clinical outcomes with one report of 17 of 18 repairs resulting in retears. ${ }^{13}$
RCR technique is determined by the pattern and size of the tear with increasing repair complexity as the size of the tear increases. In practice repair techniques adapt to individual patterns of tear. ${ }^{14,15}$ While SR to DR repair comparisons often result in similar clinical outcomes. ${ }^{7,9,16,17}$ there are fewer retears when a DR repair is used for larger tears which are more likely to result in revision surgery. ${ }^{18}$

Clear differences in outcomes are found in biomechanical studies in cadavers and animal models indicating when there is a massive RC tear that the DR repair provides greater tendon contact area and higher ultimate failure loads. Recently it has been suggested that a triple row repair would result in an improved restoration of rotator cuff anatomy for large and massive tears. ${ }^{19}$ In comparing the tendon contact pressure resulting from several repair techniques, the 'diamondback' repair had the best tendon to bone contact. ${ }^{20} \mathrm{~A}$ recent biomechanical comparison of SR and DR repairs to a novel triple row (NTR) repair of porcine infraspinatus tendon reported NTR repairs exhibited smaller gap size formation after repeated force cycling and higher ultimate failure load (UFL) than SR or DR repairs. ${ }^{21}$

A particular concern is return to work after rotator cuff repair (RCR) for workers that are often subjected to greater exposures than the general population. Repair outcomes have been reported as worse for workers' comp patients possibly due to exposure intensity, tendon tear size, and return to work expectations. ${ }^{22-26}$ This the first analysis of a retrospective Case series of worker's comp patients' RCR outcomes using a novel triple row (NTR) suture anchor compared to a Modified Mason Allen repair as measured by clinical outcomes. 


\section{Methods}

Arthroscopic rotator cuff repair (RCR) was performed on 112 workers' comp patients with either an MMA..$^{27,28}$ or a NTR repair between 2001 and 2014 by a single surgeon that did a Sports Fellowship. Procedures consisted of 59 MMA repairs (19 female, 40 male) and 53 NTR repairs (20 female, 33 male). MMA repairs varied somewhat but in general followed a mattress pattern with a rip stop. ${ }^{27}$ MMA repair employed two $5.5 \mathrm{~mm}$ polyetheretherketone (PEEK) RC anchors, single loaded with ultra-high molecular weight polyethylene (UHMWPE) suture that were inserted medially.

NTR repair consisted of two 5.5 PEEK RC double-loaded anchors inserted medially and one or two $5.5 \mathrm{~mm}$ knotless anchors inserted laterally. Initially 2 double loaded anchors are inserted in the greater tuberosity footprint. Next, a leading and trailing horizontal pass is made along the posterior and anterior tear margin. All suture tying is performed after the passing is complete. The far-far pass is made by passing 2 sutures from opposing anchors along the medial aspect of the tendon. The near-near passes is made by passing suture from opposing anchors along the lateral tear edge. The leading and trailing passes are now tied arthroscopically with sliding knots with multiple half hitches. One suture is then cut from each pass. The other free suture will be incorporated into the knotless lateral construct. The farfar sutures are then brought out the cannula and an air square knot is tied. One suture is then cut. By pulling on the near-near free sutures, the far-far pass is reduced to the footprint. The near-near passes is tied using multiple arthroscopic half hitches. Both of the sutures are then cut. The 3 leftover sutures are then incorporated into a lateral knotless construct to achieve a novel triple row repair. NTR repair procedure is illustrated in a videos for a single knotless anchor version. ${ }^{29,30}$ All anchors were molded generic PEEK anchors (RōG, Orland Park, IL).

Outcome measures are forward flexion and external rotation measured in degrees and external strength with supraspinatus isolation measured on a scale of 5. Outcomes were measured at four intervals: 1,3 , and 6 months with a final determination performed at variables times up to 40 months determined by patient availability which was constrained by workers comp status. UCLA shoulder scores were collected.

\section{Results}

The principal determinant of a RCR technique is the size and pattern of the tear. Tears were quantified by their anterior-posterior extent and their medial-lateral extent (Table 1). An additional measure of the tear area was the product of the two extents (4th row, Table 1). Possible gender bias was accounted for by separate analysis before combining the results (columns $6 \& 7$, Table 1). There is a small gender difference that lacks clinical significance permitting analysis of the combined data. Tear sizes in the NTR group were significantly larger than for the MMA repair group for all three tear measures.

Forward flexion and external rotation measures were essentially the same for MMA and NTR repairs (Table 2). Each measure exhibited clinical improvement over the course of recovery with the most of the improvement occurring in the first three months. Strength measurements were also very close in magnitude. MMA repairs had slightly higher repair strength than NTR repairs for males noting that NTR repairs were performed on larger tears. There was a mixed result for females: MMA external strength was better than NTR while supra isolation strength was better for NTR than MMA repair results. Forward and external flexion measures were modestly higher for MMA than NTR repairs at all times.

As noted, average tear size was significantly larger for repairs made with the NTR relative to MMA technique. When the results for tears $<2.1 \mathrm{~cm}^{2}$ in the NTR group are analyzed several outcome measures are improved: forward flexion, external rotation and supra isolation strength were larger at all times. When the results for males were analyzed with the tear size limitation, all outcome measures improved and several outcome measures for NTR repairs were larger than for MMA repairs. However, the differences were small and when both genders were included in the analysis outcomes were essentially the same.

Table I Tear sizes for the patient population by repair type and gender

\begin{tabular}{|c|c|c|c|c|c|c|}
\hline Tear size & MMA-M & NTR-M & MMA-F & NTR-F & MMA (M+F) & NTR (M+F) \\
\hline Anterior-Posterior (SD)cm & I.3 (0.37) & $2.14(0.72)$ & I.2I (0.25) & $\mathrm{I} .88(0.5 \mathrm{I})$ & $1.28(0.34)$ & $2.03(0.67)$ \\
\hline Range: $\mathrm{cm}$ & $\mathrm{I}-2$ & $1.5-4$ & $\mathrm{I}-\mathrm{I} .5$ & $\mathrm{I}-3.5$ & $\mathrm{I}-2$ & $\mathrm{I}-4$ \\
\hline Medial-Lateral (SD)cm & $\mathrm{I} .08(0.27)$ & $1.73(1.01)$ & I $(0.0)$ & $\mathrm{I} .53(0.83)$ & $\mathrm{I} .05(0.22)$ & $1.65(0.93)$ \\
\hline Range: $\mathrm{cm}$ & $\mathrm{I}-2.5$ & $\mathrm{I}-5$ & $1-1$ & $\mathrm{I}-4$ & $\mathrm{I}-3$ & $1-5$ \\
\hline Area $(S D) \mathrm{cm}^{2}$ & $|.4|(0.60)$ & $4.29(4.42)$ & $\mathrm{I} .2 \mathrm{I}(0.25)$ & $3.21(2.84)$ & $\mathrm{I} .35(0.52)$ & $3.87(3.95)$ \\
\hline Range: $\mathrm{cm}^{2}$ & $\mathrm{I}-3.75$ & $\mathrm{I}-20$ & $1-1.5$ & $\mathrm{I}-14$ & $\mathrm{I}-3.75$ & $\mathrm{I}-20$ \\
\hline Count & 40 & 33 & 19 & 20 & 59 & 53 \\
\hline
\end{tabular}


Table 2 Average of four measures of shoulder function for MMA and NTR $\mathrm{RCR}$ techniques

\begin{tabular}{|c|c|c|}
\hline Functional measure & $\begin{array}{l}\text { MMA } \\
\text { tear area } \\
1.35 \mathrm{~cm}^{2}\end{array}$ & $\begin{array}{l}\text { NTR } \\
\text { tear area } \\
3.87 \mathrm{~cm}^{2}\end{array}$ \\
\hline I mo. forward flexion (deg.) & 136.6 & 129.3 \\
\hline 3 mo. forward flexion (deg.) & 160 & 153.2 \\
\hline 6 mo. forward flexion (deg.) & 164 & 161.6 \\
\hline final forward flexion (deg.) & 163 & 159.9 \\
\hline I mo. external rotation (deg.) & 33.9 & 27.8 \\
\hline 3 mo. external rotation (deg.) & 45.6 & $4 I$ \\
\hline 6 mo. external rotation (deg.) & 49.6 & 47.5 \\
\hline final external rotation (deg.) & 49.8 & 47.1 \\
\hline I mo. external strength & 4.5 & 4.66 \\
\hline 3 mo. external strength & 4.85 & 4.66 \\
\hline 6 mo. external strength & 4.9 & 4.81 \\
\hline final external strength & 4.89 & 4.8 \\
\hline I mo. supra isolation strength & 4.41 & 4.36 \\
\hline 3 mo. supra isolation strength & 4.62 & 4.51 \\
\hline 6 mo. supra isolation strength & 4.7 & 4.59 \\
\hline final supra isolation strength & 4.71 & 4.59 \\
\hline
\end{tabular}

\section{Revisions}

A principal concern of rotator cuff repair is the need for revision surgery. Two surgical revisions were made on MMA repaired shoulders while 8 were made on NTR repaired shoulders. Revision rate was $3.4 \%$ for MMA repairs and $15 \%$ for NTRs. Whether the tear size difference is the sole reason for more revisions when a NTR was used cannot be determined given other variables such as fatty infiltration that contribute to the tendon either failing to heal or retearing.

\section{Discussion}

The purpose of rotator cuff repair is the restoration of the tendon footprint as a precursor of healing. Biomedical testing of SR and DR repairs concluded that DR repairs are to be preferred for large tears. $^{7,8,30-32}$ NTR repair focusses on footplate restoration. NTR repair is supported by two 'triple' row biomechanical studies that demonstrated tendon contact pressure was increased. ${ }^{19,20}$ NTR was tested biomechanically and demonstrated higher ultimate failure load and larger load cycles to failure than SR and DR repairs. ${ }^{21}$ Small gap formation under a cyclic loading paradigm (10 to $180 \mathrm{~N}$ cycled 3500 times) and high UFLs were hallmarks of NTR repair.

Clinically there are additional RCR considerations that are important. Repair complexity, number of anchors and sutures (cost), open, mini-open, arthroscopic repair and their effect on recovery time. Compared to the other two 'triple' row repair techniques, NTR repair typically used three anchors versus five anchors in the other techniques. It is rare that there is sufficient humeral head real estate to insert more than three anchors and inserting additional anchors in the humerus could compromise its integrity.

Outcome measures for RCRs using either MMA or NTR procedures were essentially the same despite the fact that tear size in the NTR group was significantly larger. When gender results were analyzed taking into account that tear size was somewhat smaller in females, the outcome measures were essentially the same for both groups and not significantly different from males thereby allowing combining the results. When the tear sizes were comparable for MMA and NTR for males, all the performance measures were improved for NTR repairs and several measures were somewhat better than for MMA repairs.

That outcome measures for the two repair techniques were similar is consistent with SR- and DR- RCR comparisons concluding that the clinical result is the same. ${ }^{6,8-10,18}$ This may reflect the difficulty of following up patient outcomes for sufficiently long times. Clinical study completion are affected by patient mobility, intervening events (shoulder trauma), cooperativeness, inaccurate recall, and aging effects that compromise study completion and accuracy. Patient satisfaction is often at a higher level than would be expected based on MRI outcomes indicating compromised repairs.

With increasing tear size the surgeon has to adjust the repair technique to accommodate the individual tear pattern and size. Increasing tear size may be correlated with poor tendon physiological condition and hence be more prone to requiring revision surgery. Retear rates for SR and DR repairs have varied from 10 to $94 \% .{ }^{1,6,9,13,33-}$ ${ }^{37}$ Many factors contribute to retear rate variability such as tendon condition, tear size, patient age, and the suggestion that RCR results for workers' comp patients are poorer than the general population. The $15 \%$ retear rate for NTR repairs of large tears in a worker's comp population is comparable or better than retear rates achieved previously.

\section{Cost consideration}

A triple repair procedure utilized 5 anchors and was in effect a single level mattress repair. ${ }^{19}$ The Diamondback repair is essentially three mattress sutures in a single row with the tendon lateral edge held down with three knotless anchors using a total of 5 anchors. ${ }^{20}$ It was shown to exhibit the best compression of the tendon to the footplate of several techniques that were tested. NTR repair has been shown to be robust for holding the tendon in place in a biomechanical study and the present results indicate that it results in acceptable clinical outcomes. ${ }^{21}$ Using the preferred three generic anchor NTR repair there is a significant cost savings: NTR repair (RoG Sports Medicine \$190, $2 \times 5.5 \mathrm{~mm}$ PEEK anchor- \$70, 5.5 knotless anchor- \$50) versus a Diamondback- 5 anchor repair using brand name anchors $\$ 2,250$ : 5 $x \$ 450$ ). In our experience there are few instances where 3 lateral anchors would be tolerated due to available bone landscape. The use of generic anchors results in a net savings of $\$ 2,060$ per case.

\section{Conclusion}

It has been suggested that 5-10year follow-up studies are needed to determine long term outcomes given that SR and DR rotator cuff repairs have similar clinical functional and subjective patient outcomes. Perhaps a more important consideration is why no clinical difference is observed. One possibility is that $\mathrm{RC}$ repairs never restore the RC to its optimal intact state irrespective of the repair technique. Suturing could compromise tendon blood supply. Genes required for repair and reattachment of the tendon to bone may be compromised, silent or absent. ${ }^{38}$ Biology plays a predominant role in healing. 
However, despite these limitations current repair techniques point to increasing the number of sutures through the tendon results in a better repair. ${ }^{39}$ Based on clinical and biomedical outcomes of NTR rotator cuff repair it is recommended that large tears be repaired with multiple suture passes through medial to lateral positions. NTR repair results in small gap formation, high ultimate failure load, and significant cost savings.

\section{Acknowledgments}

None.

\section{Conflicts of interest}

The authors declare there is no conflict of interest.

\section{Reference}

1. Sugaya H, Maeda K, Matsuki K, et al. Repair Integrity and Functional Outcome after Arthroscopic Double-Row Rotator Cuff Repair. A Prospective Outcome Study. J Bone Joint Surg Am. 2007;89(5):953-960.

2. Chung SW, Kim, JY, Kim MH, et al. Arthroscopic repair of massive rotator cuff tears: outcome and analysis of factors associated with healing failure or poor postoperative function. Am J Sports Med. 2013;41(7):1674-1683.

3. Mihalko WM, Bergin PF, Kelly FB, et al. Obesity, Orthopaedics, and Outcomes. J Am Acad Orthop Surg. 2014;22(11):683-690.

4. Mall NA, Tanaka MJ, Choi LS, et al. Factors affecting rotator cuff healing. J Bone Joint Surg Am. 2014;96(9):778-788.

5. Colvin AC, Egorova N, Harrison AK, et al. National Trends in Rotator Cuff Repair. J Bone Joint Surg Am. 2012;94(3):227-233.

6. Franceschi F, Ruzzini L, Longo UG, et al. Equivalent clinical results of arthroscopic single-row and double-row suture anchor repair for rotator cuff tears a randomized controlled trial. Am J Sports Med. 2007;35(8):1254-1260.

7. Saridakis P, Jones G. Outcomes of single-row and double-row arthroscopic rotator cuff repair: a systematic review. J Shoulder Joint Surg Am. 2010;92(3):732-742.

8. Grasso A, Milano G, Salvatore M, et al. Single-row versus double-row Artroscopic rotator cuff repair: A prospective randomized clinical study. Arthroscopy. 2009;25(1):4-12.

9. Millett PJ, Warth RJ, Dornan GJ, Lee JT, Spiegl UJ (2014) Single-row versus double-row rotator cuff repair: a systematic review and metaanalysis of level I randomized clinical trials. J Shoulder Elbow Surg 23(4): 586-597.

10. Russell RD, Knight JR, Mulligan E, Khazzam MS (2014) Structura integrity after rotator cuff repair does not correlate with patient function and pain. J Bone Joint Surg Am 96(4): 265-271.

11. McElvany MD, McGoldick E, Gee AO, et al. Rotator cuff repair: Published evidence on factors associated with repair integrity and clinical outcome. Am J Sports Med. 2014;43(2):491-500.

12. Gartsman GM, Drake G, Edwards TB, et al. Ultrasound evaluation of arthroscopic full-thickness supraspinatus rotator cuff repair: single-row versus double-row suture bridge (transosseous equivalent) fixation. Results of a prospective, randomized study. J Shoulder Elbow Surg. 2013;22(11):1480-1487.

13. Galatz LM, Ball CM, Teefey SA, et al. The outcome and repair integrity of completely arthroscopically repaired large and massive rotator cuff tears. J Bone \& Joint Surg. 2004;86-A(2):219-224.

14. DeHaan AM, Axelrad TW, Kaye E, et al. Does double-row rotator cuff repair improve outcome of patients compared with single-row technique?: A systematic review. Am J Sports Med. 2011;40(5):11761185.

15. Noticewala MS, Ahmad CS. Double-row rotator cuff repair: The new gold standard. Tech Shoulder Elbow Surg. 2015;16(1):6-9.

16. Prasathaporn N, Kuptniratsaikul S, Kongrukgreatiyos K. Single-row repair versus double-row repair of full-thickness rotator cuff tears. Arthroscopy. 2011;27(7):978-985.

17. Nho SJ, Slabaugh MA, Seroyer ST, et al. Does the literature support double-row suture anchor fixation for arthroscopic rotator cuff repair? A systematic review comparing double-row and single-row suture anchor configuration. Arthroscopy. 2009;25(11):1319-13320.

18. Duquin TR, Buyea C, Bisson LJ. Which method of rotator cuff repair leads to the highest rate of structural healing? A systematic review. Am J Sports Med. 2010;38(4):835-841.

19. Ostrander RV, Andrews J. Arthroscopic triple-row rotator cuff repair: A modified suture-bridge technique. Orthopedics. 2009;32(8):566-570.

20. Burkhart SS, Denard PJ, Obopilwe E, et al. Optimizing pressurized contact area in rotator cuff repair: the diamondback repair. Arthroscopy. 2012;28(2):188-195

21. Rhode BA, Rhode WS. Biomechanical comparison of single- doubleand novel triple- row rotator cuff repairs in a porcine model using generic anchors. MOJ Orthop Rheumatol. 2015;2(4):1-5.

22. Misamore GW, Ziegler DW, Rushton JL. Repair of the rotator cuff. A comparison of results in two populations of patients. J Bone Joint Surg Am. 1995;77(9):1335-1339.

23. Watson EM, Sonnabend DH. Outcome of rotator cuff repair. $J$ Shoulder Elbow Surg. 2002;11(3):201-211.

24. Bhatia S, Piasecki DP, Nho SJ, et al. Early return to work in workers' compensation patients after arthroscopic full-thickness rotator cuff repair. Arthroscopy. 2010;26(8):1027-1034.

25. Namdari S, Donegan RP, Chamberlain AM, et al. Factors affecting outcome after structural failure of repaired rotator cuff tears. $J$ Bone Joint Surg Am. 2014;96(2):99-105.

26. Henn RF, Tashjian RZ, Kang L, et al. Patients with workers' compensation claims have worse outcomes after rotator cuff repair. J Bone Joint Surg Am. 2008;90(10):2105-2113.

27. Lee BG, Cho NS, Rhee YG. Modified mason-allen suture bridge technique: a new suture bridge technique with improved tissue holding by the modified mason-allen stitch. Clin Orthop Surg. 2012;4(3):242245 .

28. Scheibel MT, Habermeyer P. A modified mason-allen technique for rotator cuff repair using suture anchors. Arthroscopy. 2003;19(3):330333.

29. Novel Triple Row Repair procedure video.

30. GraphPadSoftware.

31. Carbonel I, Martinez AA, Calvo A, et al. Single-row versus double-row arthroscopic repair in the treatment of rotator cuff tears: a prospective randomized clinical study. Int Orthop. 2012;36(9):1877-1883.

32. Funk L, Auplish S. Simple double-row rotator cuff repair technique for large and massive cuff tears. Techniques in Shoulder \& Elbow Surg. 2009;10(1):39-42.

33. Koh KH, Kang KC, Lim TK, et al. Prospective randomized clinical trial of single- versus double-row suture anchor repair in 2- to 4-cm rotator cuff tears: clinical and magnetic resonance imaging results. Arthroscopy. 2011;27(4):453-462. 
34. Kim JR, Cho YS, Ryu KJ, et al. Clinical and radiographic outcomes after arthroscopic repair of massive rotator cuff tears using a suture bridge technique assessment of repair integrity on magnetic resonance imaging. Am J Sports Med. 2012;40(4):786-793.

35. Gartsman GM, Drake G, Edwards TB, et al. Ultrasound evaluation of arthroscopic full-thickness supraspinatus rotator cuff repair: single-row versus double-row suture bridge (transosseous equivalent) fixation. Results of a prospective, randomized study. J Shoulder Elbow Surg. 2013;22(11):1480-1487.

36. Charousset C, Grimberg J, Duranthon LD, et al. Can a double-row anchorage technique improve tendon healing in arthroscopic rotator cuff repair? Am J Sports Med. 2007;35(8):1247-1253.
37. Burks RT, Presson AP, Weng HY. An analysis of technical aspects of the arthroscopic bankart procedure as performed in the united states. Arthroscopy. 2014;30(10):1246-1253.

38. Galatz L, Rothermich S, VanderPloeg K, et al. Development of the supraspinatus tendon-to-bone insertion: localized expression of extracellular matrix and growth factor genes. $J$ Orthop Res. 2007;25(12):1621-1628.

39. Bicos J, Mazzocca AD, Hallab N, et al. The multi-suture technique for rotator cuff repair: a biomechanical evaluation. Orthopedics. 2007;30(11):910-919. 